\title{
Article \\ Aroma Profiles of Dry-Hopped Ciders Produced with Citra, Galaxy, and Mosaic Hops
}

\author{
Matthew T. Bingman ${ }^{1}$, Josephine L. Hinkley ${ }^{2}$, Colin P. Bradley II ${ }^{3}$ and Callie A. Cole ${ }^{4, *}$ \\ 1 Department of Chemistry \& Biochemistry, University of Oregon, 1585 E 13th Ave., Eugene, OR 97403, USA; \\ mbingman@uoregon.edu \\ 2 Rocky Vista College of Osteopathic Medicine, 255 E Center St., Ivins, UT 84738, USA; \\ josephine.hinkley@ut.rvu.edu \\ 3 Department of Chemistry, Columbia Basin College, 2600 N 20th Ave., Pasco, WA 99301, USA; \\ cbradley@columbiabasin.edu \\ 4 Department of Chemistry \& Biochemistry, Fort Lewis College, 1000 Rim Drive, Durango, CO 81301, USA \\ * Correspondence: ccole@fortlewis.edu
}

check for updates

Citation: Bingman, M.T.; Hinkley, J.L.; Bradley, C.P., II; Cole, C.A. Aroma Profiles of Dry-Hopped Ciders Produced with Citra, Galaxy, and Mosaic Hops. Appl. Sci. 2022, 12, 310. https://doi.org/10.3390/ app12010310

Academic Editor: Agata Górska

Received: 24 November 2021

Accepted: 20 December 2021

Published: 29 December 2021

Publisher's Note: MDPI stays neutral with regard to jurisdictional claims in published maps and institutional affiliations.

Copyright: (c) 2021 by the authors. Licensee MDPI, Basel, Switzerland. This article is an open access article distributed under the terms and conditions of the Creative Commons Attribution (CC BY) license (https:// creativecommons.org/licenses/by/ $4.0 /)$.

\begin{abstract}
Cider quality and consumer acceptance are greatly influenced by its aroma. With the continued expansion of the craft cider industry, cider producers are employing techniques such as dry hopping to develop unique flavor profiles. Few studies, however, have explored the VOCs of dry-hopped cider. Herein, we monitor the development of VOCs from pressed apple juice, through fermentation and dry hopping by HS-SPME-GC-MS, to elucidate when and how aroma compounds arise in cider production. In all, 89 VOCs were detected, spanning eight classes of organic compounds. Racking events decreased ester concentrations by $10 \pm 1 \%$, but resting on the lees allowed these pleasant, fruity aromas to be reestablished. Dry hopping was conducted with three types of hops (Citra, Galaxy, and Mosaic). The varied development of terpenes and esters between hop varieties supports the use of this technique to diversify the aroma profiles of ciders. Herein, we report that both the variety of hops and the timing of key processing steps including racking and hop addition significantly alter the identity and concentration of aroma-important VOCs in dry-hopped cider.
\end{abstract}

Keywords: cider; dry hopping; gas chromatography; mass spectrometry; solid phase microextraction; volatiles

\section{Introduction}

Cider consumption over the past two decades in the United States has seen substantial growth. From 2010 to 2018, consumption of specialty beverages, including cider, grew 487\%, and in 2018, represented an industry worth USD 2.2 billion in the USA [1]. Although most commercially-produced cider is still fermented from imported apple juice concentrates or processing fruits (fruit that did not make it for consumption sale) [1], the demand for beverages crafted naturally and locally is high [2]. Before Prohibition, southwest Colorado, USA, had a diverse population of apple varietals used for fresh consumption and cider production. The Montezuma Orchard Restoration Project (MORP) has documented 436 apple varietals planted in Colorado prior to 1930 [3]. MORP is working with local orchards to restore Colorado's rich history in cider production by genetically profiling apple trees across the state. With an increased understanding of which apple varietals are currently growing in the region and what organoleptic cider qualities they produce, cider makers can make informed decisions on selections of fruit to harvest and process to a final product based on measurable chemical characteristics. This work focuses on the Gravenstein apple which is common to southwest Colorado and is considered a quality cider apple [4,5]. Results reported herein have been added to the MORP cider chemistry database to inform cider makers about the properties of southwest Colorado cider apples.

With increased cider consumption and production, cider makers are looking for ways to diversify their products. One such approach that is gaining traction is the use 
of hops within the cider-making process. Dry hopping is a technique that is commonly used in the beer brewing industry [6]. This process involves the addition of whole cone hops or dried hop pellets to the beverage after fermentation has been completed. Dry hopping is desirable in the brewing industry, because the hops impart appealing aroma compounds without the bitterness or astringency typically associated with hops. Although dry hopping has been well studied within the beer brewing industry, little work has been carried out to understand how dry hopping impacts the aroma profile of cider. Hops have been shown to contain numerous aroma compounds which include esters, ketones, short-chain fatty acids, and terpenes [7]. Previous studies have shown that the three most prominent "woody" terpenes [8] in hops, comprising up to $80 \%$ of the total volatile organic compounds (VOCs), are $\beta$-myrcene, caryophyllene, and humulene [7]. The story is not quite this simple, however. The VOCs that dominate the headspace of hops are often quite different from those in the headspace of dry-hopped beer, due to biotransformation and other variables involved in the fermentation process $[9,10]$. For this reason, it is important to monitor the aroma development after dry hopping, to differentiate which aroma active compounds were efficiently extracted from the hops into the beverage, and how they may have changed. In this work, we identify when and to what extent these and other aroma-important species, outlined in Figure 1, are incorporated into the cider headspace during cider making and over the dry hopping process. These previous studies suggest that not only the variety of hops, but the timing of key processing steps including racking and hop addition, significantly alter the identity and concentration of aroma-important VOCs in fermented beverages.

\section{A. Internal Standards}

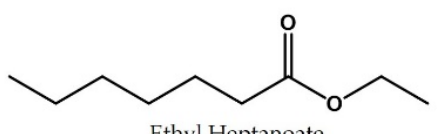

\section{B. Cider VOCs}
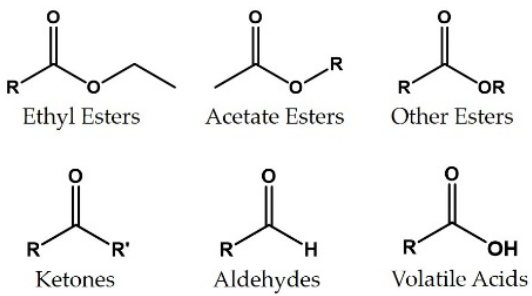

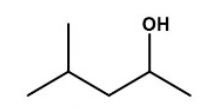

4-Methyl-2-Propanol

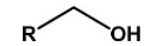

Higher Alcohols
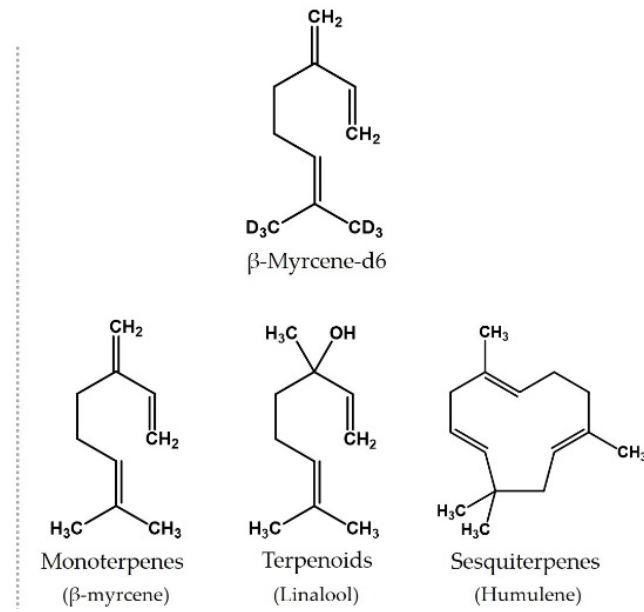

Figure 1. The structures of internal standards (A) and key cider volatile organic compounds of importance to this study (B).

Volatile compounds in cider have been successfully analyzed by sampling the headspace above the beverage [11]. Headspace-solid phase micro extraction-gas chromatographymass spectrometry (HS-SPME-GC-MS) offers a simple and robust approach to analyze cider VOCs that has been used extensively in recent publications [11-15]. Headspace sampling requires a SPME fiber that will efficiently preconcentrate volatiles of interest. Due to the large variety of compounds in a cider matrix, a divinyl-benzene/carboxen/ polydimethylsiloxane (DVB/CAR/PDMS) fiber was chosen for this study to select for compounds with varying degrees of polarity. Importantly, this study does not focus on thiols, which can be an important contributor to hopped beverage aroma [16]. These species may be sampled more efficiently by a different SPME fiber or sorptive extraction method than the method that we report herein.

Chemical analysis of dry-hopped cider is severely lacking in the published literature. We seek to fill this knowledge gap by monitoring the evolution of VOCs in the headspace of a novel, single varietal (Gravenstein) apple juice inoculated with a popular strain of 
saccharomyces cervisiae (Lalvin QA23), to perform three replicate and parallel fermentations. By measuring the VOC development throughout the 135-day fermentation and maturation, we elucidate how key processing steps such as racking influence the development of cider aroma over time. After fermentation completes, three different varieties of hops (Citra, Mosaic, and Galaxy) are added to the three ciders (one hop varietal per fermentation), to examine how the dry hopping process creates aroma differentiation among otherwise identical cider samples over the course of 8 days. Herein, we test the hypothesis that both hop variety and the timing of cider processing events (racking and dry hopping) are central contributors to final cider aroma development.

\section{Materials and Methods}

\subsection{Apple Fruit Collection}

Gravenstein apples were handpicked from a small, privately-owned orchard in Hermosa, Colorado. Fruit was picked from six different trees that have been genetically profiled as the Gravenstein varietal by MORP (Tree ID: ca2c010) [17] and thus used in this study for apple varietal consistency. Fruit was only collected if free of damage and visible disease. Flesh softness and skin color (ground color) observations were used to determine apple ripeness, and both orchard owners and local cider makers (EsoTerra Cider, Dolores, CO, USA) were consulted to verify consistency across apple types. The fruit was stored in a temperature-controlled $\left(1.7^{\circ} \mathrm{C}\right)$ environment immediately after collection for two and one half weeks to await further processing.

\subsection{Apple Fruit Processing and Juice Analysis}

Whole Gravenstein apples were rinsed and ground using an electric fruit mill, and then pressed into a juice with a hand press. Fruit was washed with dechlorinated tap water and ground and pressed at room temperature. One week prior to total sample processing, a small preliminary sample of Gravenstein apples was juiced and analyzed for tannin concentration $(0.0467 \% \pm 0.4)$, titratable acidity $(0.770 \% \pm 0.008$ malic acid equivalents), specific gravity $(1.049 \pm 0.005)$, and $\mathrm{pH}(3.28 \pm 0.02)$, as these are pre-fermentation parameters important to the cider-making process.

Specific gravity and $\mathrm{pH}$ were also measured before sampling the apple juice for HSSPME-GC-MS analysis. All measurements were conducted in triplicate. The limit of detection (LOD) and limit of quantitation (LOQ) values were calculated based on the ASBC method for low-level detection [18]. LOD is defined as LOD $=\bar{x}_{B}+3 \sigma$ and LOQ is defined as $\mathrm{LOQ}=\bar{x}_{B}+10 \sigma\left(\bar{x}_{B}=\right.$ mean signal of replicate reagent blanks, $\mathrm{N}=10$; and $\sigma=$ standard deviation of the blank). The reported precision of our method is one standard deviation of the three trials.

Tannin concentration and titratable acidity were determined by the well-established Lowenthal permanganate titration and the malic acid titration, respectively [4]. The Lowenthal permanganate titration was carried out by adding $1 \mathrm{~g}$ of indigo carmine (Millipore Sigma, Saint Louis, MO, USA, 85\%) and $50 \mathrm{~mL}$ concentrated sulfuric acid (Fisher Scientific, Waltham, MA, USA, $98.0 \%$ ) to $1000 \mathrm{~mL}$ of deionized water. A total of $5 \mathrm{~mL}$ of the indigo carmine solution and $1 \mathrm{~mL}$ of Gravenstein apple juice were then added to $150 \mathrm{~mL}$ of deionized water. An indicator blank was prepared by combining $5 \mathrm{~mL}$ indigo carmine solution and $150 \mathrm{~mL}$ of deionized water. These solutions were titrated with $0.005 \mathrm{M}$ potassium permanganate (Millipore Sigma, ACS Reagent, $\geq 99.0 \%$ ) until a golden yellow end point was reached. The malic acid titration was performed by diluting $25 \mathrm{~mL}$ of Gravenstein apple juice to $100 \mathrm{~mL}$ with deionized water and titrating with $0.2 \mathrm{M} \mathrm{NaOH}$ (Fisher Scientific, $\geq 97.0 \%$ ) until a visible end point was reached. Phenol red indicator (Millipore Sigma, ACS reagent) was added to observe the color change. The concentration of malic acid equivalents was calculated according to the published expression by Miles et al. [5].

A Vernier PH-BTA $\mathrm{pH}$ probe was used throughout this study to measure the $\mathrm{pH}$ of juice and cider. Before each measurement, it was calibrated with buffers of known $\mathrm{pH}$. Specific gravity was determined using an EasyDens portable density meter from Anton 
Paar. In addition to the juice analysis, both measurements were taken with every sample throughout fermentation.

\subsection{Fermentation}

The bulk batch of Gravenstein apples were milled and pressed at room temperature. Juice was collected in three $18.9 \mathrm{~L}$ glass carboys for simultaneous triplicate fermentations. Each carboy was inoculated with $5.00 \pm 0.03 \mathrm{~g}$ of dry Lalvin QA23 Saccharomyces cerevisiae yeast that was reconstituted with filtered water $\left(35-40^{\circ} \mathrm{C}\right)$ and then immediately capped with airlocks. Carboys were then transferred to a dark, $14.4 \pm 1.7^{\circ} \mathrm{C}$ temperature-controlled chamber for fermentation. Approximately $20 \mathrm{~mL}$ aliquots from each fermentation vessel were used for HS-SPME-GC-MS analysis, $\mathrm{pH}$, and specific gravity readings every $48 \mathrm{~h}$ for 22 days. After 22 days, samples were monitored once a week until all specific gravities plateaued (47 days). On day 25 of alcoholic fermentation, the cider was cold-crashed ( $24 \mathrm{~h}$ at $2{ }^{\circ} \mathrm{C}$ ) and racked.

\subsection{Dry Hopping}

After 135 days of fermentation and maturation, the three ciders were racked into six 11.4 L carboys (two per fermentation: one control sample and one dry-hopped sample). Totals of $7 \mathrm{~g}$ of Galaxy, Citra, and Mosaic hop pellets (Artisan Hops) were added to 3 of the ciders (one hop varietal per cider). This ratio of hops to cider volume was recommended on the Artisan Hops packaging. Ciders were kept in contact with the hops for eight days at $14.4 \pm 1.7^{\circ} \mathrm{C}$, a common temperature range for fermentation and maturation [19]. HSSPME-GC-MS samples were taken on day 5 and day 8 of dry hopping and analyzed for VOC development. Control samples that were not dry hopped were sampled on day eight for comparison.

\subsection{Headspace-Solid Phase Microextraction (HS-SPME)}

Cider sample aliquots (10 mL), $2.6 \mathrm{~g}$ of $\mathrm{NaCl}$ (Sigma Aldrich, St. Louis, MO, USA), and internal standards were transferred to $20 \mathrm{~mL}, 22.5 \times 75 \mathrm{~mm}$ glass headspace vials (Sigma Aldrich) for solid phase microextraction (SPME). A volume of $30 \mu \mathrm{L}$ of 4-methyl-2-propanol $\left(0.80 \mathrm{~g} \mathrm{~mL}^{-1}\right)$ and $1 \mu \mathrm{L}$ ethyl heptanoate $\left(0.87 \mathrm{~g} \mathrm{~mL}^{-1}\right)$ were added as internal standards (Millipore Sigma, 99\% and 98\%, respectively). Terpenes were semi-quantified by spiking $0.1 \mu \mathrm{L}$ of $0.01 \mathrm{~g} \mathrm{~mL}^{-1} \beta$-myrcene-d6 in ethyl acetate (Axios Research, Washington, DC, USA) [20] into the cider samples after dry hopping. The structures of the internal standards are displayed in Figure 1A and the 8 classes of compounds that were semi-quantified by these internal standards are shown in Figure 1B. Ethyl hepatanoate was used for semiquantification of ethyl esters, acetate esters, other esters, ketones, aldehydes, and volatile acids. 4-Methyl-2-propanol was used for higher alcohol semi-quantification. B-Myrcene-d6 was used to semi-quantify three classes of terpenes that developed after the addition of hops (monoterpenes, terpenoids, and sesquiterpenes). Vials were then capped with $20 \mathrm{~mm}$ aluminum release seal PTFE/silicone liner cap (Sigma Aldrich) with a manual vial crimper. Vials were held in a $40{ }^{\circ} \mathrm{C}$ water bath for ten minutes for headspace equilibration. A conditioned DVB/CAR/PDMS 50/30 $\mu \mathrm{m}$ SPME fiber (Supelco, Bellefonte, PA, USA) was then exposed to the headspace for $30 \mathrm{~min}$ for volatile preconcentration. Samples were analyzed by GC-MS within $24 \mathrm{~h}$ of exposure and stored at $4{ }^{\circ} \mathrm{C}$ in the interim. SPME fibers were conditioned after every sample according to manufacturer recommendations.

\subsection{Gas Chromatography-Mass Spectrometry (GC-MS) Analysis}

An Agilent Technologies 7820A Gas Chromatograph (GC) and a 5977E Mass Spectrometer Detector (MSD) with Mass Hunter GC-MS acquisition Software (B.07.00.1203 Agilent, Santa Clara, CA, USA) were used for all analyses. SPME fibers were introduced to the GC inlet at $260^{\circ} \mathrm{C}$ for $1 \mathrm{~min}$ in splitless mode to thermally desorb analytes and introduce them onto the column. Helium gas was used as the carrier gas at a flow rate of $1 \mathrm{~mL} \mathrm{~min}{ }^{-1}$ through an Agilent DB-5, $30 \mathrm{~m} \times 0.25 \mathrm{~mm} \times 0.25 \mu \mathrm{m}$ column. The oven was held at $50{ }^{\circ} \mathrm{C}$ 
for $5 \mathrm{~min}$, increased to $190{ }^{\circ} \mathrm{C}$ at a rate of $3{ }^{\circ} \mathrm{C} \mathrm{min}^{-1}$, increased again to $230{ }^{\circ} \mathrm{C}$ at a rate of $70{ }^{\circ} \mathrm{C} \min ^{-1}$, and then held at $230{ }^{\circ} \mathrm{C}$ for $5 \mathrm{~min}$. The transfer line to the MS was held at $230{ }^{\circ} \mathrm{C}$.

Eluates were ionized and fragmented in a $70 \mathrm{eV}$ electron ionization (EI) source and separated with a quadrupole mass spectrometer set to a mass range of $m / z 50-550$. The quadrupole interface was held at $150^{\circ} \mathrm{C}$. As Figure 2 illustrates, the deuterated internal standard ( $\beta$-myrcene-d6) was distinguished from the non-deuterated $\beta$-myrcene in the dry-hopped cider samples by selecting for $m / z 75$ and $m / z 69$, respectively. These ions likely form via $\mathrm{C}_{5} \mathrm{H}_{7}$ loss from their corresponding molecular ions, and their structures are predicted in Figure 2. Identification of analytes was accomplished by referencing the National Institute of Standards and Technology (NIST) library (Version 2.2. Agilent Technologies, Santa Clara, CA, USA) of EI spectra. Column and fiber blanks were run regularly, and siloxanes originating from the column and SPME fiber were identified and removed from the analysis [21].

A.

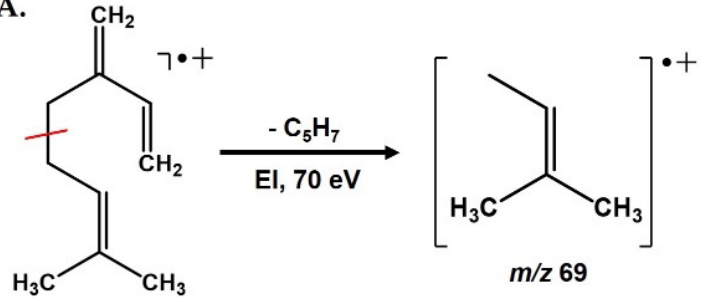

B.

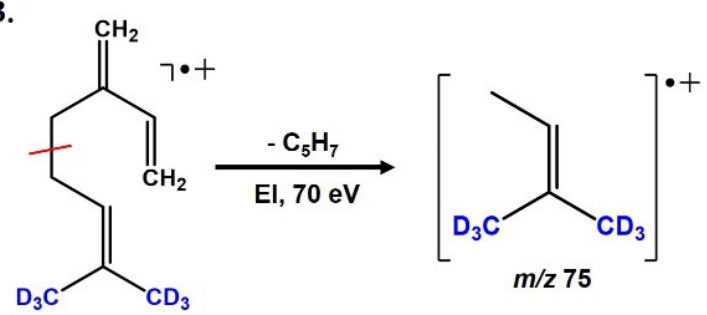

Figure 2. The electron ionization fragmentation observed for the molecular ions of $\beta$-myrcene (A) and $\beta$-myrcene-d6 (B).

\section{Results and Discussion}

\subsection{Fermentation Monitoring}

Cider makers monitor the progression of fermentation to inform their decisions regarding the timing of racking events. For the three ciders monitored in this study, specific gravity (SG) was measured with an Anton Parr EasyDens digital gravity and alcohol tester. SG and pH were measured every $48 \mathrm{~h}$ throughout fermentation and again at the end of maturation. Between the end of alcoholic fermentation and maturation, the three ciders were not sampled to reduce the potential for contamination. As Figure 3 depicts, an overall increase in $\mathrm{pH}(3.28-3.72)$ and a decrease in SG from 1.049 (juice) to 0.996 (cider) was observed, as expected. After the cider was racked (day 25), specific gravity continued to drop, and the $\mathrm{pH}$ of the ciders rose slightly to 3.51. The end of primary fermentation was on day 47 when the averaged specific gravity was $\leq 1.003$ and the average $\mathrm{pH}$ was 3.56. The three ciders were not racked through maturation and as a result rested on the remaining lees for 88 days to increase the concentration of fruity, volatile esters. During maturation, the specific gravity decreased from 1.003 to 0.996 on day 135 . The $\mathrm{pH}$ increased from 3.51 to 3.70 during this same time frame. This increase in $\mathrm{pH}$ is due to the production of ethanol throughout fermentation, which has been shown to increase the dissociation of weak acids present in the cider [22]. Similarly, the SG results were consistent with a successful fermentation. As the yeast consumes the sugars present, the density of the solution decreases, and SG values below 1.000 indicate that the fermentable sugars have been converted to ethanol and other fermentation products. 


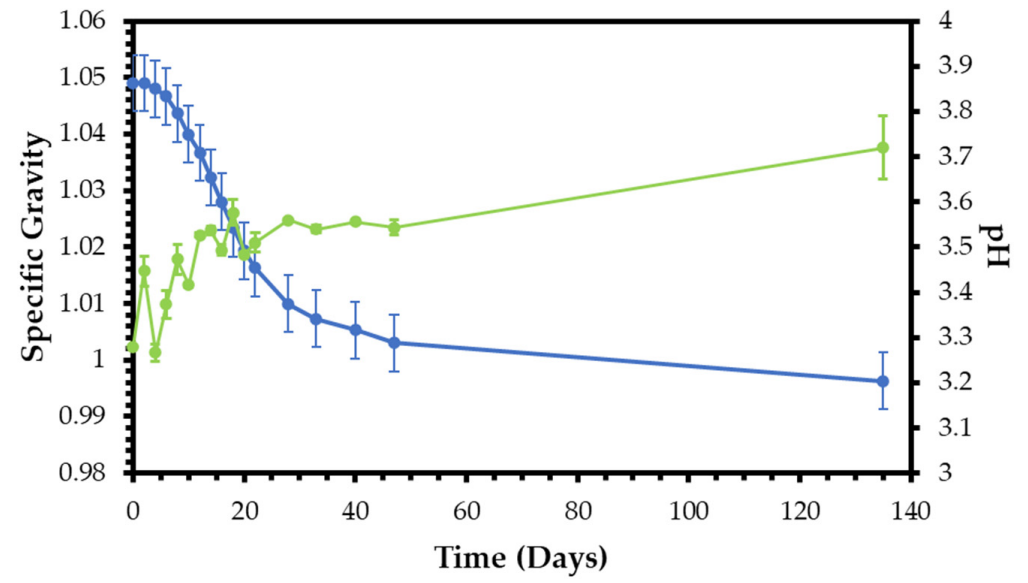

Figure 3. Specific gravity (blue) and $\mathrm{pH}$ (green) are shown for the entire fermentation and maturation process. On the left y-axis, the average specific gravity of three Gravenstein apple cider fermentations is plotted against time. The right $\mathrm{y}$-axis shows $\mathrm{pH}$ measurements for the fermentations. Day 0 corresponds to measurements taken of pre-inoculated apple juice in triplicate and Day 135 represents the end of cider maturation (prior to dry hopping).

\subsection{Higher Alcohol and Ester Analysis}

Three concurrent trials of Gravenstein apple juice were fermented to a finished cider product and monitored for changes in VOC concentrations over the course of 135 days. The chemical composition of the headspace of these ciders changed dramatically over this time frame as a result of the changing byproducts of yeast metabolism. In total, 89 VOCs were identified by HS-SPME-GC-MS throughout fermentation and maturation: 11 higher alcohols, 7 acetate esters, 10 ethyl esters, 32 other esters, 5 aldehydes, 8 ketones, 5 volatile acids, and 11 terpenes. The details of these detections are included in Supplementary Table S1. Five esters and four higher alcohols that were consistently measured above the LOQ throughout fermentation have published odor perception thresholds $[14,23]$ that were used to display the long running trends of ester and higher alcohol development throughout fermentation and maturation in both Table 1 and Figure 4.

\section{A. Esters}

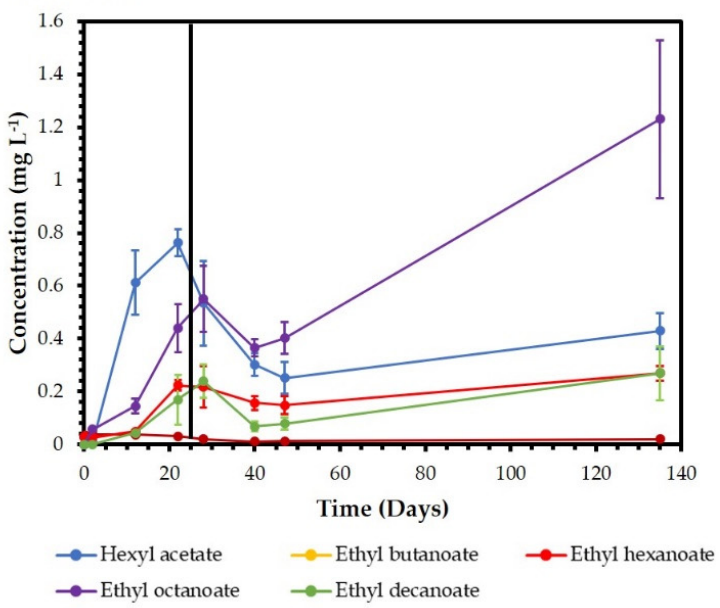

B. Higher Alcohols

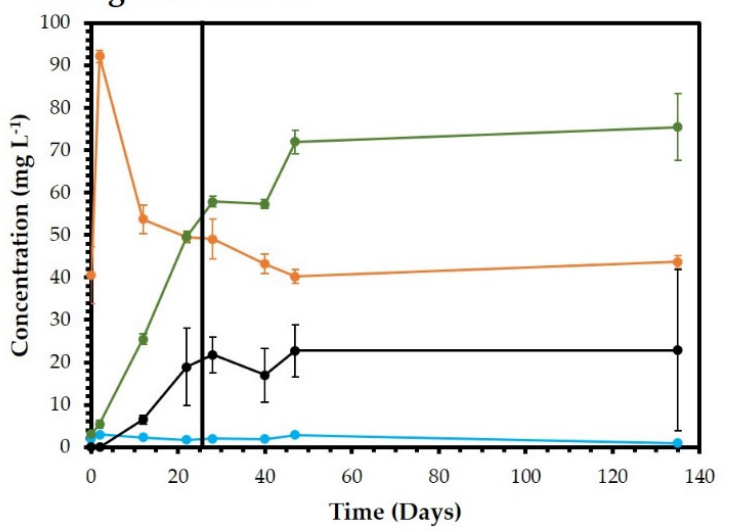

$\rightarrow$-butanol $\rightarrow$ - -hexanol $\rightarrow$-phenethyl alcohol $\rightarrow$ - -pentanol

Figure 4. HS-SPME-GCMS measurements of headspace ester (A) and higher alcohol (B) concentrations that represent the overall trends observed from juice (day 0) to mature cider (day 135). Day 25 (vertical black line) is the date of cold crashing and racking the cider samples. Error bars and are reported as $\bar{x} \pm 1 \mathrm{~s}$ of triplicate samples.

A comparison of these esters and alcohols is provided in Table $1 \mathrm{a}, \mathrm{b}$ in order of their chromatographic retention times (RT), along with their published odor descriptors and per- 
ception thresholds (PT). Concentrations of four alcohols (1-butanol, 1-propanol, 1-hexanol, and 2-phenethylethanol) and five esters (ethyl butanoate, ethyl hexanoate, hexyl acetate, ethyl octanoate, and ethyl decanoate) are shown for the 22-day period before racking (Table 1a) and the period after racking until the end of maturation (Table 1b). Prior to day 12 , higher alcohols, primarily originating from the juice, dominated the volatile composition of the ciders. By day 12 of fermentation, esters had risen significantly in concentration. In fact, from juice to cider (135 days post inoculation), total ester concentration increased by a factor of 71 , whereas the total higher alcohol concentration increased by only a factor of 3 . This agrees with the literature, as the esterification of higher alcohols efficiently occurs in fermentations with saccharomyces cerevisiae [24]. A practical insight for cider makers comes from a direct comparison from day 22 (pre-racking) to day 28 (post-racking). The total concentration of higher alcohols increased by $14 \pm 1 \%$, and the total concentration of esters decreased by $10 \pm 1 \%$ across this 6-day period. Previous research indicates that esters (including ethyl hexanoate and ethyl octanoate) are influenced by the time of contact with lees [25], but this is the first time to our knowledge that a quantifiable decrease in ester concentration has been linked directly to a racking event in cider making. Similarly, the simultaneous increase in higher alcohol concentration after racking highlights the importance of this process in tuning the cider aroma. As Table 1 shows, many of the major higher alcohols contribute floral green notes, whereas the esters are primarily perceived as fruity. These data suggest that in the days immediately following a racking event, the cider aroma may lose fruity notes. If allowed to rest on the lees for longer time periods without racking, the fruity esters have a chance to grow in.

Table 1. (a) Representative higher alcohol and ester concentrations $\left(\mathrm{mg} \mathrm{L}^{-1}\right)$ from pre-inoculation through racking. (b) Representative higher alcohol and ester concentrations $\left(\mathrm{mg} \mathrm{L}^{-1}\right)$ from racking to the end of maturation.

\begin{tabular}{|c|c|c|c|c|c|c|c|}
\hline \multicolumn{8}{|c|}{ (a) } \\
\hline Higher Alcohols & $\begin{array}{c}\mathrm{RT} \\
(\mathrm{min})\end{array}$ & $\begin{array}{c}\text { Juice } \\
\text { Concentration }^{\mathrm{a}}\end{array}$ & $\begin{array}{c}\text { Day } 2 \\
\text { Concentration }\end{array}$ & $\begin{array}{c}\text { Day } 12 \\
\text { Concentration }\end{array}$ & $\begin{array}{c}\text { Day } 22 \\
\text { Concentration }\end{array}$ & Odor Descriptor & PT $\left(\mathrm{mg} \mathrm{L}^{-1}\right)$ \\
\hline 1-butanol & 2.3 & $1.920 \pm 0.286$ & $3.008 \pm 0.358$ & $2.300 \pm 0.295$ & $1.767 \pm 0.021$ & Medicinal $^{\mathrm{b}}$ & $150^{b}$ \\
\hline 1-pentanol ${ }^{b}$ & 3.0 & $3.070 \pm 0.723$ & $5.452 \pm 0.934$ & $25.477 \pm 1.275$ & $49.65 \pm 1.367$ & Alcohol, fruity ${ }^{c}$ & $0.030-45^{c}$ \\
\hline 1-hexanol & 6.6 & $40.533 \pm 6.665$ & $92.153 \pm 1.417$ & $53.733 \pm 3.387$ & $49.433 \pm 1.037$ & Flower, green e & $8^{d}$ \\
\hline 2-phenylethanol & 17.7 & - & - & $6.500 \pm 1.019$ & $18.927 \pm 9.112$ & Floral, rose ${ }^{\mathrm{e}}$ & $14^{\mathrm{d}}$ \\
\hline \multicolumn{8}{|l|}{ Esters } \\
\hline Ethyl butanoate & 4.3 & $0.028 \pm 0.017$ & $0.039 \pm 0.008$ & $0.038 \pm 0.006$ & $0.031 \pm 0.002$ & Fruity, sweet ${ }^{\mathrm{e}}$ & $0.4^{b}$ \\
\hline Ethyl hexanoate & 12.3 & $0.031 \pm 0.018$ & $0.027 \pm 0.007$ & $0.050 \pm 0.001$ & $0.224 \pm 0.021$ & Fruity, apple ${ }^{\mathrm{e}}$ & $0.014^{\mathrm{d}}$ \\
\hline Hexyl acetate & 12.9 & - & - & $0.612 \pm 0.121$ & $0.763 \pm 0.050$ & Fruity, herb ${ }^{\mathrm{e}}$ & $0.67^{\mathrm{b}}$ \\
\hline Ethyl octanoate & 22.0 & - & $0.057 \pm 0.005$ & $0.146 \pm 0.029$ & $0.440 \pm 0.091$ & Fruity, fatty ${ }^{\mathrm{e}}$ & $0.005^{\mathrm{d}}$ \\
\hline Ethyl decanoate & 30.8 & - & - & (b) $0.044 \pm 0.013$ & $0.170 \pm 0.094$ & Fruity, grape ${ }^{b, e}$ & $0.2^{b}$ \\
\hline & & Day 28 & Day 40 & Day 47 & Day 135 & & \\
\hline Higher Alcohols & $\begin{array}{c}\text { RT } \\
(\min )\end{array}$ & Concentration $^{a}$ & Concentration & Concentration & Concentration & Odor Descriptor & PT $\left(\mathrm{mg} \mathrm{L}^{-1}\right)$ \\
\hline 1-butanol & 2.3 & $2.030 \pm 0.135$ & $1.953 \pm 0.132$ & $2.870 \pm 0.305$ & $0.930 \pm 0.268$ & Medicinal $^{b}$ & $150^{\mathrm{b}}$ \\
\hline 1-pentanol ${ }^{b}$ & 3.0 & $57.923 \pm 1.207$ & $57.350 \pm 1.084$ & $71.967 \pm 2.764$ & $75.452 \pm 7.844$ & Alcohol, fruity ${ }^{c}$ & $0.030-45^{c}$ \\
\hline 1-hexanol & 6.6 & $49.100 \pm 1.037$ & $43.167 \pm 4.691$ & $40.267 \pm 2.301$ & $43.633 \pm 1.584$ & Flower, green $\mathrm{e}$ & $8^{d}$ \\
\hline $\begin{array}{l}\text { 2-phenylethanol } \\
\text { Esters }\end{array}$ & 17.7 & $21.670 \pm 4.229$ & $17.000 \pm 6.318$ & $22.700 \pm 6.239$ & $22.857 \pm 19.067$ & Floral, rose $\mathrm{e}^{\mathrm{e}}$ & $14^{\mathrm{d}}$ \\
\hline Ethyl butanoate & 4.3 & $0.021 \pm 0.011$ & $0.012 \pm 0.002$ & $0.013 \pm 0.003$ & $0.020 \pm 0.003$ & Fruity, sweet ${ }^{\mathrm{e}}$ & $0.4^{b}$ \\
\hline Ethyl hexanoate & 12.3 & $0.218 \pm 0.078$ & $0.157 \pm 0.025$ & $0.149 \pm 0.034$ & $0.270 \pm 0.028$ & Fruity, apple $\mathrm{e}^{\mathrm{e}}$ & $0.014^{\mathrm{d}}$ \\
\hline Hexyl acetate & 12.9 & $0.534 \pm 0.161$ & $0.302 \pm 0.043$ & $0.251 \pm 0.060$ & $0.430 \pm 0.068$ & Fruity, herb ${ }^{\mathrm{e}}$ & $0.67^{b}$ \\
\hline Ethyl octanoate & 22.0 & $0.550 \pm 0.124$ & $0.365 \pm 0.032$ & $0.403 \pm 0.061$ & $1.230 \pm 0.229$ & Fruity, fatty e & $0.005^{\mathrm{d}}$ \\
\hline Ethyl decanoate & 30.8 & $0.239 \pm 0.018$ & $0.069 \pm 0.001$ & $0.079 \pm 0.023$ & $0.270 \pm 0.102$ & Fruity, grape ${ }^{b, e}$ & $0.2^{b}$ \\
\hline
\end{tabular}

${ }^{a}$ All semi-quantified analyte concentrations are reported as $\bar{x} \pm 1 \mathrm{~s} .{ }^{\mathrm{b}}[26]^{\mathrm{c}}$ 1-pentanol (balsamic, fruity, $\mathrm{PT}=0.47 \mathrm{mg} \mathrm{L}^{-1}$ ) coeluted with 3-methyl-1-butanol (alcohol, PT $=45 \mathrm{mg} \mathrm{L}^{-1}$ ) and 2-methyl-1-butanol (banana, green, $\left.\mathrm{PT}=0.030 \mathrm{mg} \mathrm{L}^{-1}\right)[24,27,28]$. A sum of these three unresolved species is reported here. ${ }^{\mathrm{d}}$ [29] ${ }^{\mathrm{e}}$ [14]. 
Figure $4 \mathrm{~A}$ depicts the ester development from juice to the end of maturation for the five esters described previously. Ethyl butanoate (fruity [24]) and ethyl hexanoate (green apple, brandy, fruity [24]) were present in the apple juice, but their concentrations increased by $26 \%$ and $38 \%$, respectively, by day 12 of fermentation. Similarly, hexyl acetate (fruity, sweet [30]), ethyl octanoate (fruity, candy, pineapple [11]), and ethyl decanoate (brandy, fruity [24]), which were not detected in the juice, were established as major species within the headspace by fermentation day 12 . Maximum concentrations of ethyl hexanoate $\left(0.224 \pm 0.021 \mathrm{mg} \mathrm{L}^{-1}\right)$ and hexyl acetate $\left(0.763 \pm 0.05 \mathrm{mg} \mathrm{L}^{-1}\right)$ during the fermentation phase were observed on day 22. Before the ciders were cold crashed and racked on day 25 (marked in Figure 4 with a vertical black line), ethyl octanoate and ethyl decanoate reached fermentation concentration maxima of $0.55 \pm 0.12 \mathrm{mg} \mathrm{L}^{-1}$ and $0.55 \pm 0.124 \mathrm{mg} \mathrm{L}^{-1}$, which are above or within error of the published perception thresholds for these species [11]. All five esters experienced a sizeable decrease to minimum concentrations following the racking event on day 25 . On day 40 , ethyl butanoate, ethyl octanoate, and ethyl decanoate had diminished by $69 \%, 33 \%$, and $71 \%$, respectively. Ethyl hexanoate and hexyl acetate decreased by $33 \%$ and $67 \%$ from their maximum concentrations by day 47 . HS-SPMEGC-MS analysis was conducted again at the end of maturation on day 135. At the end of maturation, ester concentrations increased, most notably ethyl octanoate and ethyl decanoate. This experimental evidence supports previous observations of ester growth when cider is held over yeast lees for extended periods of time. All five of these esters have a predominately fruity aroma and were all detected above perception thresholds and likely contribute pleasant fruity notes to the ciders [14].

The development and changes in four higher alcohols are shown in Figure 4B. 1Pentanol (balsamic, fruity [24]) coelutes with two other species (3-methyl-1-butanol and 2-methyl-1-butanol) that were not baseline resolved with our GC method. The integrated peak area of the three chromatographic peaks were summed and reported together as 1-pentanol. These species together were quantified to be, perhaps unsurprisingly, at the highest concentration of any other analyte observed in this study. 1-pentanol and 2-methyl1-butanol were both detected in the juice, whereas 3-methyl-1-butanol was first detected within the first 12 days of fermentation indicating the possibility of either isomerization or the development of a new fermentation byproduct. Better separation of the species could be achieved by beginning the separation at a lower temperature $\left(<50^{\circ} \mathrm{C}\right)$ or slowing the temperature ramping.

Trends for the four higher alcohols monitored over the 135-day process were much more varied than the esters. 1-Hexanol (green, herbaceous [24]) was the predominant alcohol in the juice and two days after fermentation had increased in concentration by $56 \%$. Interestingly, by day 12, 1-hexanol decreased by $42 \%$. From day 12 to the end of maturation (day 135), 1-hexanol concentration decreased another 19\%, but maintained its position as the second most abundant higher alcohol. 1-Butanol remained relatively constant throughout the 135-day process, at a concentration considerably below the perception threshold. 2-Phenylethyl alcohol (floral, honey-like [11,14]) was not present in the juice but was the third most abundant higher alcohol by the end of maturation. Although a rise was observed for many higher alcohols after racking (day 25), the alcoholic and floral aroma imparted by these species remained steady once the specific gravity reached levels close to 1.001 (day 47).

\subsection{Dry-Hopped Cider Analysis}

Each Gravenstein apple cider was dry hopped with a different variety of pressed pellet hop: Citra, Galaxy, and Mosaic. HS-SPME-GC-MS analysis was conducted after samples were in contact with the various hops for five days and eight days. This timeline was chosen because it falls within the published industry standard of 4-12 days for dry-hopping beer. Figure 5 shows the experimental chromatograms labelled with selected VOCs that were consistently observed across all samples. As illustrated by these experimental data, after only 5 days, a wide array of new VOCs is already present in the cider headspace. From 
day 5 to day 8 (3-day period) of dry hopping, total ester concentrations in Citra and Galaxy doubled. Over the same time span, total ester concentrations increased by a factor of 18 for Mosaic hops. As expected, the Mosaic hops sample also had the highest overall concentration of esters of all samples studied after 8 days of dry hopping. Esterification of hop-derived monocarboxylic acids with ethanol is likely the synthetic route to many of these volatile esters during dry hopping [6]. Terpenes, however, were added to the cider headspace most efficiently by Citra hops. Total terpene concentration doubled in the Citra sample from day 5 to day 8, whereas a negligible increase was observed for both Galaxy and Mosaic hopped samples. Not only did Citra hops yield the highest concentration of terpenes after 8 days, they also generated the largest variety of unique terpenes at concentrations greater than the LOQ. More investigation into the timeline for dry hopping cider is needed; however, these preliminary results suggest that dry hopping time could be shorter than many current beer industry practices, and added VOCs are heavily dependent on hop type.
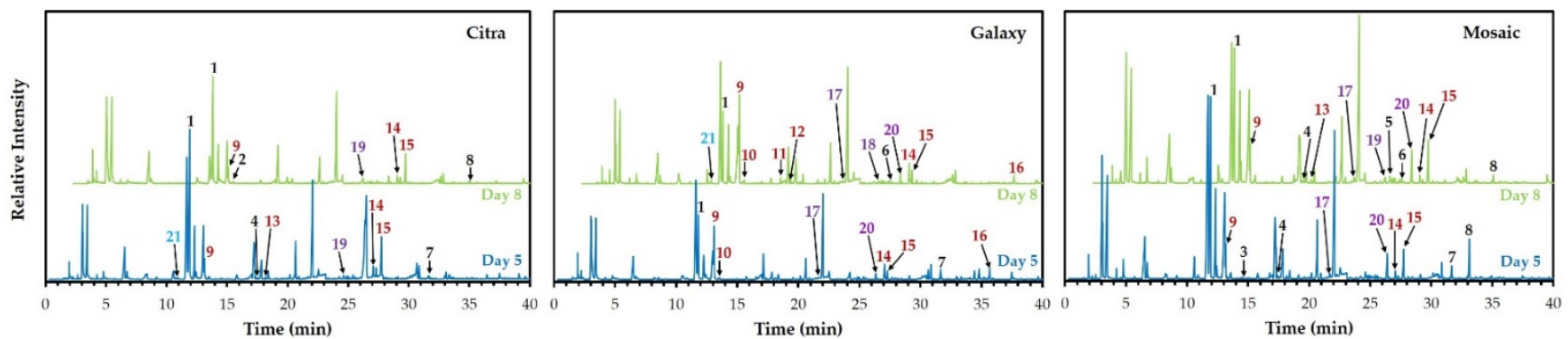

Figure 5. HS-SPME-GC-MS chromatograms are shown after 5 (blue) and 8 (green) days of dry hopping with three hop varieties (Citra, Galaxy, and Mosaic). Select VOCs that originated from the hops and were consistently detected across samples are numbered here. Terpenes are in black 1-8 (1: $\beta$-myrcene; 2: D-limonene; 3: ocimene; 4: linalool; 5: geraniol; 6: cyclotene; 7: caryophyllene; 8: humulene), esters are denoted in red 9-16 (9: 3-methylbutyl-2-methylpropanoate, 10: methyl heptanoate, 11: methyl nonanoate, 12: 2-ethylhexyl pentanoate, 13: 2-methylbutyl-2-methylbutanoate, 14: methyl 4-decanoate, 15: methyl geranoate, 16: 2-methyl propanoate), ketones are purple 17-20 (17: 2-dodecanone, 18: 2-tridecanone, 19: 2-decanone, 20: 2-undecanone) and propanoic acid is light blue (21).

Although no sensory work was conducted for this study, many relevant perception threshold analyses have been previously published for the VOCs observed in these experiments. We have summarized those VOCs and their previously published aromas here. Supplementary Table S2 includes every hop-derived VOC with published odor descriptors and perception thresholds. For a complete list of all detected species during the dry hopping process, please refer to Supplementary Table S1. Five VOCs were semi-quantified above published perception thresholds in these samples, as shown in bold in Supplementary Table S2: $\beta$-myrcene, linalool, methyl heptanoate, 2-nonanone, and propanoic acid. $\beta$-Myrcene (citrus, spicy $[23,31]$ ) was the only terpene found in all 6 hopped samples, and was also present at the highest concentration of all the terpenes. As its name suggests, Citra hops produced the highest concentration of this citrusy terpene. $\beta$-Myrcene has also been identified as the predominant aroma compound in other hop varieties [8]. Linalool (citrus, floral $[23,31]$ ) was also semi-quantified above the perception threshold in both Citra- and Mosaic-hopped samples, but was not generated from Galaxy hops in detectable quantities. Previously established as one of the most important hop-derived odorants in dry hopped beer [6], linalool also contributes greatly to dry-hopped cider aroma. Overall, citrus aroma-contributors were much more concentrated in Citra- and Mosaic-hopped samples when compared with those hopped with Galaxy. Rather, Galaxy hops created more fruity VOCs, as evidenced by methyl heptanoate existing in quantifiable abundance only in this hop variety (fruity [32]). One floral ketone, 2-nonanone, was semi-quantified above the 
perception threshold in Mosaic hopped samples only. Lastly, propanoic acid may add a spicy, soy fragrance in ciders hopped with Citra and Galaxy. Each hop variety generated a unique set of aroma-contributing VOCs when added to identical cider samples: Citrahopped cider was citrus and spicy, Galaxy-hopped cider was fruity, and Mosaic-hopped cider had both citrus and floral VOCs based on these perception threshold analyses.

\section{Conclusions}

Quantitative analysis of triplicate Gravenstein apple cider fermentations has revealed a large array of VOCs. The 89 total VOC detected by HS-SPME-GC-MS throughout fermentation and dry hopping included higher alcohols (11), acetate esters (7), ethyl esters (10), other esters (32), aldehydes (5), ketones (8), volatile acids (5), and terpenes (11). Specifically, species above the published perception thresholds were monitored carefully, revealing three unique ciders with appealing citrus (Citra hops), fruity (Galaxy hops), and floral (Mosaic hops) aromas. During the fermentation process, esters develop quickly, but drop in concentration ( $-10 \%)$ immediately following racking events. This is of practical interest to cider makers when planning the timeline of racking and bottling. By contrast, a single cold crash and racking event increased higher alcohol concentrations ( $+14 \%)$ for 3 days afterwards. Although few studies have focused on dry hopping in a cider context, we hypothesized based on previous beer publications that not only the variety of hops, but the timing of hop addition significantly alter the identity and concentration of aromaimportant VOCs in cider. This hypothesis was confirmed. Citra hops added the highest concentration of terpenes in the cider headspace, and Mosaic hops added the highest concentration of esters. From day 5 to day 8, a significant increase in ester concentration was observed for all hop varieties. Although further research is needed to fully characterize the dry hopping process for cider making through detailed sensory analysis, this work provides novel analytical insight into how cider makers might make informed decisions on the application of hops to diversify the aroma profile of their beverages. Similarly, we have provided useful information on racking events and insight into how a single varietal apple cider's aroma profile changes throughout fermentation, maturation, and dry hopping. Relevant data discussed in this work have been added to the MORP cider chemistry database to inform cider makers about the properties of southwest Colorado cider apples.

There are myriad variables involved in cider making, all of which are worthy of further exploration. Important future work should include optimizing the dry hopping process to limit the quantity of hops used while maximizing desirable aroma contributors, thereby decreasing cost for cider makers and decreasing the environmental impact of excessive hop use.

Supplementary Materials: The following are available online at https://www.mdpi.com/article/10 .3390/app12010310/s1, Table S1: Semi-quantification of volatile compounds (mg/L) in juice, cider, and dry-hopped cider and Table S2: Semi-quantified VOCs a $\left(\mathrm{mg} \mathrm{L}^{-1}\right)$ added to cider headspace as a result of dry hopping for 5 and 8 days with Citra, Galaxy, and Mosaic hops.

Author Contributions: C.A.C., undergraduate students M.T.B. and J.L.H., and collaborator C.P.B.II implemented the study and interpreted the results together. Undergraduate students M.T.B. and J.L.H. collected and analyzed all reported experimental data under the mentorship of C.A.C. and C.P.B.II, M.T.B. prepared the manuscript draft and all manuscript editing was performed by C.A.C. C.A.C. acquired all funding that supported this work and led project administration and supervision. All authors have read and agreed to the published version of the manuscript.

Funding: This work was made possible thanks to the 2019-2020 Undergraduate Analytical Research Program (UARP) grant of the Society for the Analytical Chemists of Pittsburgh (SACP) and the 2019-2020 American Society for Mass Spectrometry Research at PUIs Award funded by Agilent Technologies. We thank these organizations for their financial support of undergraduate research. 
Data Availability Statement: Results of this work have been reported in the publicly available Montezuma Orchard Restoration Project (MORP) database linked below and can also be provided by the corresponding author upon request. https:/ / airtable.com/shrixCDelEJbp0SrV/tblUWhAm7 Mtbh6QUJ/viwEQfYwX XwQJISdEa (accessed on 1 June 2021).

Acknowledgments: We thank Jared Scott, Elizabeth Philbrick, and Avery Scott of EsoTerra Cider, for their collaborative insights, cider samples, and assistance picking and pressing the apples used in this study. We also thank them greatly for the uses of their facilities for sample preparation. Special thanks go to the orchard owners in Hermosa, CO for their Gravenstein apples. We acknowledge and thank Sam Bingman, Marty Emmes, Kelly Bleck, Zach Myers, and Michael Grubb for their discussions, edits, advice, and assistance throughout the project. Lastly, our laboratory would not function without the continual support of several good dogs, cats, and rabbits: thank you especially to O'Malley, Liessel, Tilli, Ivy, and Stuart for your continual support and cuteness.

Conflicts of Interest: The authors declare no conflict of interest.

\section{References}

1. Miles, C.A.; Alexander, T.R.; Peck, G.; Galinato, S.P.; Gottschalk, C.; Van Nocker, S. Growing Apples for Hard Cider Production in the United States-Trends and Research Opportunities. HortTechnology 2020, 30, 148-155. [CrossRef]

2. Watson, B. 2020 Points and 2021 Predictions. Available online: https://www.brewersassociation.org/insights/2020-points-and2021-predictions / (accessed on 25 June 2021).

3. Schuenemeyer, A.; Schuenemeyer, J. Montezuma Orchard Resoration Project. Available online: https://montezumaorchard.org/ (accessed on 24 May 2021).

4. Lafontaine, S.R.; Shellhammer, T.H. Impact of static dry-hopping rate on the sensory and analytical profiles of beer. J. Inst. Brew. 2018, 124, 434-442. [CrossRef]

5. Miles, C.A.; King, J.; Alexander, T.; Scheenstra, E. Evaluation of Flower, Fruit, and Juice Characteristics of a Multinational Collection of Cider Apple Cultivars Grown in the U.S. Pacific Northwest. HortTechnology 2017, 27, 431-439. [CrossRef]

6. Brendel, S.; Hofmann, T.; Granvogl, M. Dry-Hopping to Modify the Aroma of Alcohol-Free Beer on a Molecular Level-Loss and Transfer of Odor-Active Compounds. J. Agric. Food Chem. 2020, 68, 8602-8612. [CrossRef] [PubMed]

7. Duarte, L.M.; Amorim, T.L.; Grazul, R.M.; de Oliveira, M.A.L. Differentiation of aromatic, bittering and dual-purpose commercial hops from their terpenic profiles: An approach involving batch extraction, GC-MS and multivariate analysis. Food Res. Int. 2020, 138, 109768. [CrossRef] [PubMed]

8. Van Opstaele, F.; De Causmaecker, B.; Aerts, G.; De Cooman, L. Characterization of Novel Varietal Floral Hop Aromas by Headspace Solid Phase Microextraction and Gas Chromatography-Mass Spectrometry/Olfactometry. J. Agric. Food Chem. 2012, 60, 12270-12281. [CrossRef]

9. Rettberg, N.; Biendl, M.; Garbe, L.-A. Hop Aroma and Hoppy Beer Flavor: Chemical Backgrounds and Analytical Tools-A Review. J. Am. Soc. Brew. Chem. 2018, 76, 1-20. [CrossRef]

10. Werrie, P.-Y.; Deckers, S.; Fauconnier, M.-L. Brief Insight into the Underestimated Role of Hop Amylases on Beer Aroma Profiles. J. Am. Soc. Brew. Chem. 2021, 1-9. [CrossRef]

11. Bingman, M.T.; Stellick, C.E.; Pelkey, J.P.; Scott, J.M.; Cole, C.A. Monitoring Cider Aroma Development throughout the Fermentation Process by Headspace Solid Phase Microextraction (HS-SPME) Gas Chromatography-Mass Spectrometry (GC-MS) Analysis. Beverages 2020, 6, 40. [CrossRef]

12. Perestrelo, R.; Silva, C.L.; da Silva, P.M.C.; Medina, S.; Pereira, R.; Câmara, J.S. Untargeted fingerprinting of cider volatiles from different geographical regions by HS-SPME/GC-MS. Microchem. J. 2019, 148, 643-651. [CrossRef]

13. Nešpor, J.; Karabín, M.; Štulíková, K.; Dostálek, P. An HS-SPME-GC-MS Method for Profiling Volatile Compounds as Related to Technology Used in Cider Production. Molecules 2019, 24, 2117. [CrossRef] [PubMed]

14. Englezos, V.; Torchio, F.; Cravero, F.; Marengo, F.; Giacosa, S.; Gerbi, V.; Rantsiou, K.; Rolle, L.; Cocolin, L. Aroma profile and composition of Barbera wines obtained by mixed fermentations of Starmerella bacillaris (synonym Candida zemplinina) and Saccharomyces cerevisiae. LWT 2016, 73, 567-575. [CrossRef]

15. Wei, J.; Zhang, Y.; Qiu, Y.; Guo, H.; Ju, H.; Wang, Y.; Yuan, Y.; Yue, T. Chemical composition, sensorial properties, and aroma-active compounds of ciders fermented with Hanseniaspora osmophila and Torulaspora quercuum in co- and sequential fermentations. Food Chem. 2020, 306, 125623. [CrossRef]

16. Ochiai, N.; Sasamoto, K.; Kishimoto, T. Development of a Method for the Quantitation of Three Thiols in Beer, Hop, and Wort Samples by Stir Bar Sorptive Extraction with in Situ Derivatization and Thermal Desorption-Gas Chromatography-Tandem Mass Spectrometry. J. Agric. Food Chem. 2015, 63, 6698-6706. [CrossRef] [PubMed]

17. Schuenemeyer, A.; Schuenemeyer, J. Montezuma Orchard Restoration Project Map. Available online: https://morp-acp.maps. arcgis.com/apps / webappviewer / index.html?id=91f531fb0d9843fab5d7455c15addefd\&extent=-12208863.2258\%2C4278369.278 6\%2C-11132629.8676\%2C5105112.1766\%2C102100 (accessed on 24 May 2021).

18. ASBC. ASBC Methods of Analysis, 14th ed.; ASBC: St. Paul, MN, USA, 2011. 
19. Herrero, M.; García, L.A.; Díaz, M. Volatile Compounds in Cider: Inoculation Time and Fermentation Temperature Effects. J. Inst. Brew. 2006, 112, 210-214. [CrossRef]

20. Dennenlöhr, J.; Thörner, S.; Manowski, A.; Rettberg, N. Analysis of Selected Hop Aroma Compounds in Commercial Lager and Craft Beers Using HS-SPME-GC-MS/MS. J. Am. Soc. Brew. Chem. 2019, 78, 16-31. [CrossRef]

21. Curran, A.M.; Rabin, S.I.; Prada, P.A.; Furton, K.G. Comparison of the Volatile Organic Compounds Present in Human Odor Using Spme-GC/MS. J. Chem. Ecol. 2005, 31, 1607-1619. [CrossRef]

22. Akin, H.; Brandam, C.; Meyer, X.-M.; Strehaiano, P. A model for $\mathrm{pH}$ determination during alcoholic fermentation of a grape must by Saccharomyces cerevisiae. Chem. Eng. Process. Process. Intensif. 2008, 47, 1986-1993. [CrossRef]

23. Miyazawa, M.; Nagata, T.; Nakahashi, H.; Takahashi, T. Characteristic odor components of essential oil from Caesalpinia decapetala. J. Essent. Oil Res. 2012, 24, 441-446. [CrossRef]

24. He, W.; Liu, S.; Heponiemi, P.; Heinonen, M.; Marsol-Vall, A.; Ma, X.; Yang, B.; Laaksonen, O. Effect of Saccharomyces cerevisiae and Schizosaccharomyces pombe strains on chemical composition and sensory quality of ciders made from Finnish apple cultivars. Food Chem. 2021, 345, 128833. [CrossRef]

25. Antón-Díaz, M.J.; Valles, B.S.; Hevia, A.G.; Lobo, A.P. Aromatic Profile of Ciders by Chemical Quantitative, Gas ChromatographyOlfactometry, and Sensory Analysis. J. Food Sci. 2014, 79, S92-S99. [CrossRef]

26. Arcari, S.; Caliari, V.; Sganzerla, M.; Godoy, H.T. Volatile composition of Merlot red wine and its contribution to the aroma: Optimization and validation of analytical method. Talanta 2017, 174, 752-766. [CrossRef]

27. Yu, H.; Xie, T.; Xie, J.; Chen, C.; Ai, L.; Tian, H. Aroma perceptual interactions of benzaldehyde, furfural, and vanillin and their effects on the descriptor intensities of Huangjiu. Food Res. Int. 2020, 129, 108808. [CrossRef]

28. Tura, D.; Failla, O.; Bassi, D.; Pedò, S.; Serraiocco, A. Cultivar influence on virgin olive (Olea europea L.) oil flavor based on aromatic compounds and sensorial profile. Sci. Hortic. 2008, 118, 139-148. [CrossRef]

29. Escudero, A.; Gogorza, B.; Melús, M.A.; Ortín, N.; Cacho, A.J.; Ferreira, V. Characterization of the Aroma of a Wine from Maccabeo. Key Role Played by Compounds with Low Odor Activity Values. J. Agric. Food Chem. 2004, 52, 3516-3524. [CrossRef] [PubMed]

30. Aparicio, R.; Morales, M.T. Characterization of Olive Ripeness by Green Aroma Compounds of Virgin Olive Oil. J. Agric. Food Chem. 1998, 46, 1116-1122. [CrossRef]

31. Wijaya, C.H.; Hadiprodjo, I.T.; Apriyantono, A. Identification of Volatile Compounds and Key Aroma Compounds of Andaliman Fruit (Zanthoxylum Acanthopodium DC.). Food Sci. Biotechnol. 2002, 2, 680-683.

32. Pino, J.A.; Trujillo, R. Characterization of odour-active compounds of sour guava (Psidium acidum [DC.]Landrum) fruit by gas chromatography-olfactometry and odour activity value. Flavour Fragr. J. 2021, 36, 207-212. [CrossRef] 